\title{
Kidney, Pancreas and Islet Transplant Options for Patients with Diabetic Nephropathy
}

\section{Costas Fourtounas* and Periklis Dousdampanis}

Department of Internal Medicine-Nephrology, Patras University Hospital, Patras, Greece

\begin{abstract}
Recent progress in surgery and immunosuppression has expanded the "transplant menu" for patients with diabetic nephropathy which is now including: Kidney Transplantation from a Deceased Donor (DDKT) or a Living Donor (LDKT), Simultaneous Pancreas Kidney (SPK), Pancreas Transplantation Alone (PTA), Pancreas after Kidney (PAK) and Islet transplantation. As pre-emptive transplantation presents a clear survival advantage over dialysis, all diabetic patients with chronic kidney disease (CKD) should be referred for early evaluation by a transplant center. For type 1 diabetes mellitus (T1DM) patients, LDKT and SPK transplantation offer superior and approximately equivalent long-term patient and allograft survival. PAK transplant rates tend to decline due to surgical and immunological complications, but it may still be considered for well selected candidates with preserved kidney allograft function.

For type 2 diabetes mellitus (T2DM) patients, not only LDKT, but even DDKT are superior to dialysis. SPK transplantation should be offered only in selected cases with special metabolic characteristics similar to T1DM. PTA should be considered only for selected cases of T1DM with well preserved renal function (eGFR>80 ml/min $/ 1.73 \mathrm{~m}^{2}$ and minimal proteinuria), as it may a cause of rapid deterioration of renal function. Islet transplantation should still be considered as an experimental procedure for T1DM, and has no place in patients with advanced CKD, but it may be applied in already immunosuppressed patients following KT. However, the best transplant option for patients with diabetic nephropathy therapy should always be individualized, taking under consideration the patients' preferences and expectations, their overall medical condition and the transplant center's experience with all these procedures.
\end{abstract}

Keywords: Diabetes; Diabetic nephropathy; Islet transplantation; Kidney transplantation; Pancreas transplantation

\section{Introduction}

Diabetes Mellitus (DM) is a worldwide epidemic and the leading cause of Chronic Kidney Disease (CKD), blindness, stroke, heart attack and amputations [1,2]. Type $1 \mathrm{DM}(\mathrm{T} 1 \mathrm{DM})$ is an autoimmune disease leading into $\beta$ cells destruction, whereas type $2 \mathrm{DM}$ (T2DM) is a chronic degenerative disease mainly due to insulin resistance. About $30-40 \%$ of patients with DM will eventually present a gradual deterioration of their renal function accompanied by proteinuria and retinopathy and will be classified with the diagnosis of diabetic nephropathy. Currently, about $40 \%$ of the CKD patients starting renal replacement therapies (hemodialysis or peritoneal dialysis) carry this diagnosis and present increased morbidity and mortality compared with other causes of CKD.

Several years ago, diabetic nephropathy had been considered as a relative or absolute contraindication for renal transplantation, due to cardiovascular and infectious complications and unacceptable morbidity and mortality. However, the landmark study of Wolfe et al. [3] has shown that renal transplantation provided a clear survival advantage for diabetics with End-Stage Renal Disease (ESRD) and reduced mortality by $73 \%$ compared with patients remaining on the waiting list. The projected life expectancy was more profound for younger diabetics (presumably T1DM) reaching a gain of 17 years, whereas the gain was only 3 years for patients older than 60 years (presumably T2DM).

Historically, the first successful pancreas transplantation was done in 1966 in the US in order to cure patients with T1DM [4]. However, pancreas transplantation was not fostered so easily by the transplant community due to increased rates of surgical complications and rejections of the pancreatic allografts. Gradual progress in surgical techniques and the advent of more powerful immunosuppressive agents has improved significantly the initial outcomes [5-10] and by the end of 2010 more than 35000 pancreas transplantations have been totally reported to the International Pancreas Transplant Registry (IPTR) with the vast majority (24000) performed in the US [11].

The first successful case of allogeneic islet transplantation in humans with T1DM was reported in 1990 by Scharp et al. [12]. However, the procedure did not become popular until the Edmonton study in 2000 [13] which showed insulin independence in seven T1DM patients with a steroid free regimen.

So, the potential therapies for patients with diabetes have expanded beyond insulin and may target normoglycemia more aggressively without the fears of hypoglycemia. The diabetic patient has now more options (pancreas transplantation, kidney transplantation, islet transplantation), but has to undergo a major operation (solid organ transplantation) and to be long-life immunosuppressed with all the possible consequences. As pancreas or islets transplantation is not a life-saving procedure and there are no clear indications, not only the patients with diabetes but also the physicians may become confused about optimal solutions for diabetic nephropathy (Table 1).

*Corresponding author: Costas Fourtounas, Department of Internal MedicineNephrology, Patras University Hospital, Rio-Patras, 26500, Greece, Tel: +30 2610999366; Fax: +30 2610999724; E-mail: cfourt@usa.net

Received February 19, 2013; Accepted March 16, 2013; Published March 21 2013

Citation: Fourtounas C, Dousdampanis P (2013) Kidney, Pancreas and Isle Transplant Options for Patients with Diabetic Nephropathy. J Diabetes Metab S9: 001. doi:10.4172/2155-6156.S9-001

Copyright: (c) 2013 Fourtounas C, et al. This is an open-access article distributed under the terms of the Creative Commons Attribution License, which permits unrestricted use, distribution, and reproduction in any medium, provided the original author and source are credited. 


\begin{tabular}{|c|c|c|c|c|}
\hline Transplant Type & Donor Type & Indications for Diabetes Mellitus & Advantages & Disadvantages \\
\hline $\begin{array}{c}\text { Pancreas Transplantation Alone } \\
\text { (PTA) }\end{array}$ & $\begin{array}{l}\text { Deceased } \\
\text { or } \\
\text { Living (rarely) }\end{array}$ & T1DM with preserved renal function & $\begin{array}{l}\text { Short- and long-term outcomes } \\
\qquad \uparrow \uparrow \uparrow\end{array}$ & $\begin{array}{l}\text { - Peri-operative complications } \uparrow \uparrow \\
\text { - Life-long immunosuppression } \uparrow \uparrow \uparrow\end{array}$ \\
\hline Kidney Transplantation (KT) & $\begin{array}{l}\text { Deceased } \\
\text { or } \\
\text { Living }\end{array}$ & T1DM and T2DM with CKD stage 4-5 & $\begin{array}{l}\text { Short- and long-term outcomes } \\
\qquad \uparrow \uparrow \uparrow\end{array}$ & $\begin{array}{c}\text { - Peri-operative complications } \uparrow \\
\text { - No impact on diabetes control } \\
\text { - Life-long immunosuppression } \uparrow \uparrow \uparrow\end{array}$ \\
\hline $\begin{array}{l}\text { Simultaneous Pancreas Kidney } \\
\text { (SPK) }\end{array}$ & $\begin{array}{l}\text { Deceased } \\
\text { or } \\
\text { Living (rarely) }\end{array}$ & $\begin{array}{l}\text { T1DM and T2DM (by indications) with CKD } \\
\text { stage } 4-5\end{array}$ & \begin{tabular}{|c|} 
- Short- and long-term outcomes \\
$\uparrow \uparrow \uparrow$ \\
- Excellent diabetes control \\
- Single operation
\end{tabular} & $\begin{array}{l}\text { - Peri-operative complications } \uparrow \uparrow \\
\text { - Life-long immunosuppression } \uparrow \uparrow \uparrow\end{array}$ \\
\hline Pancreas After Kidney (PAK) & Deceased & T1DM with a kidney allograft & $\begin{array}{l}\text { - Short- and long-term outcomes } \\
\qquad \uparrow \uparrow \\
\text { - Excellent diabetes control }\end{array}$ & $\begin{array}{l}\text { - Peri-operative complications } \uparrow \uparrow \\
\text { - Two consecutive operations } \\
\text { - Life-long immunosuppression } \uparrow \uparrow \uparrow\end{array}$ \\
\hline Islet Transplantation & Deceased & $\begin{array}{l}\text { T1DM with preserved renal function } \\
\text { or } \\
\text { with a kidney allograft }\end{array}$ & $\begin{array}{c}\text { - no operation } \\
\text { - Peri-operative complications } \\
\downarrow \downarrow \downarrow\end{array}$ & $\begin{array}{c}\text { - Short- and long-term outcomes } \downarrow \\
\text { or } \downarrow \downarrow \\
\text { - Life-long immunosuppression } \uparrow \uparrow \uparrow\end{array}$ \\
\hline
\end{tabular}

PTA: Pancreas Transplantation Alone; KT: Kidney Transplantation; SPK: Simultaneous Pancreas Kidney; PAK: Pancreas After Kidney; IT: Islet transplantation for patients with type 1 diabetes mellitus (T1DM) or type 2 diabetes mellitus (T2DM) (CKD: Chronic Kidney Disease)

Table 1: Transplant options.

\section{Pancreas Transplantation Alone (PTA)}

PTA refers to the transplantation of a pancreas transplant graft alone. Its origin may be from a Deceased Donor (DDPTA) or a segmental graft by a Living Donor (LDPTA).

\section{Kidney Transplantation (KT)}

KT refers to the transplantation of a renal transplant graft alone. Its origin may be from a Deceased Donor (DDKT) or by a Living Donor (LDKT). When the operation takes place before the need of dialysis it is referred as pre-emptive KT $[14,15]$.

\section{Simultaneous Pancreas Kidney (SPK)}

SPK transplantation refers to the combined transplantation of both organs, coming usually from the same donor, during a single operation. The origin of the grafts is usually from deceased donors, but there are also reports of segmental pancreatic grafts from living donors [16].

\section{Pancreas after Kidney (PAK)}

Transplantation refers to the transplantation of a pancreas graft by a second surgery, following a successful kidney transplant (DDKT or LDKT). Obviously the two grafts come from different donors.

\section{Islet Transplantation (IT)}

IT refers to the transplantation of isolated pancreatic islets, which have been harvested from one or more deceased donors. It may be combined (although rare) with every type of kidney transplantation.

In the present review, we will try to provide essential information for physicians not involved in transplantation medicine, enlighten the grey zones and review in an unbiased way the contemporary data about transplant options for patients with diabetic nephropathy making clear separations between patients with T1DM and T2DM and patients with early and advanced CKD.

\section{Surgical Procedures for Organ or Islet Transplantation}

Kidney transplantation (KT) in diabetic patients has no essential differences compared with the traditional transplant procedure in non diabetic patients with ESRD. Briefly, the renal allograft is placed in the lower right or left abdomen and the renal vessels (artery and vein) are usually anastomosed with the iliac artery and vein of the recipient. The donor's ureter is anastomosed with the recipient's bladder.
Islet transplantation (IT) is not a classic surgical procedure and the islets are percutaneously infused into the portal vein [13].

Pancreas transplantation remains a challenging operation, even after $>40$ years of experience and no surgical technique has achieved universal experience $[5,6,10,11,17]$. Usually, a whole pancreaticoduodenal graft is used in cadaveric transplantation. Vascular supply derives from the right iliac artery and vein (systemic drainage). The initial expectations about a possible extra benefit by drainage to the portal venous circulation have not been fulfilled and this type of drainage is used in about $10-20 \%$ of the operations. Pancreatic exocrine secretions can be drained via the recipient's bladder or enterically (recipient's small bowel or duodenum). Although bladder drainage was the original and most practiced procedure in the past, enteric drainage is now used in the majority of the transplant centers (90\%) around the world [11]. Bladder drainage has the advantage of measuring urinary amylase as a marker of allograft function or rejection, but is accompanied by urinary tract infections, erosive cystitis, hematuria, severe metabolic acidosis and hypovolemia $[5,10,11,17,18]$. Recently, laparoscopic [19] and robotic surgery [20] have also been applied for pancreas transplantation with very encouraging results. Finally, partial pancreatic explant from living donors has been documented with excellent results in experienced transplant centers [16,21-25].

Simultaneous Pancreas Kidney transplantation (SPK) is a combination of both transplant procedures by putting the pancreas graft in the right side and the kidney graft in the left side due to anatomical reasons. The transplant surgeon proceeds with the pancreatic allograft first and then the kidney is anatomized with the left iliac vessels.

\section{Donor Evaluation for Kidney and Pancreas Transplan- tation}

Donor evaluation is crucial for KT, SPK and PAK transplantation and donor demographics have a significant impact on recipient outcomes [26,27]. However, organ shortage and increased demand for transplantation around the world have made transplant teams to expand the criteria for deceased donors' selection for kidney transplantation [28].

Regarding PTA and SPK from deceased donors, traditionally only healthy young adults (age $<40$ years) with trauma associated brain death, short stay in the ICU, plasma amylase no greater than three time the 
normal values and no abdominal trauma have been considered suitable for pancreas donation $[18,29]$. According to a recent report from the IPTR only $6 \%$ of the deceased donors were older than 45 years and only $4 \%$ of pancreas donations were from donors after cardiac death [11]. The final decision usually relies on the allograft's natural appearance at the time of organ retrieval by the transplant team. Regarding HLA matching, current trends focus mainly on the quality of the grafts and more than $50 \%$ of pancreatic transplantations are performed with 5 or 6 mismatches [11].

Regarding IT transplantation, pancreata from one or more donors that are not suitable for PTA or SPK are usually retrieved and transferred to the special transplant center which is equipped with the infrastructure for islet isolation.

Finally, the scarce of organs for transplantation has made feasible living related pancreas donation [23-25]. However, this policy has been restricted mainly to experienced transplant centers in the US, Europe and Japan. Donor hemipancreatectomy may lead to decreased serum insulin levels and obesity should be considered as an absolute contraindication for living donation. According to the Minnesota experience, pancreas donation presents minimal perioperative complications, but some donors $(<5 \%)$ may become insulin depended in the long run [16].

\section{Pre-transplant Risk Evaluation in Patients with Diabetic Nephropathy}

The goal of the pre-transplant risk evaluation is to determine whether the candidate is eligible for transplantation and discuss the potential options for KT, SPK or PAK.

The contraindications are the same as for any other organ transplantation including the presence of malignancy, active infection, psychiatric disease, drug/alcohol dependence, morbid obesity and untreated or end-stage organ damage with special emphasis on cardiovascular comorbidities [30,31]. Age should not be considered as an absolute contraindication for KT [32], but most transplant centers do not accept diabetic patients older than 45-50 years for SPK or PAK [11]. This is mainly due to an increased rate of surgical complications and lower graft survival rates [8-10], although some other studies did not confirm these results [33].

Obesity $\left(\mathrm{BMI}>30 \mathrm{~kg} / \mathrm{m}^{2}\right)$ is also considered as a relative contraindication for transplantation in diabetic patients as it is accompanied with inferior outcomes for both KT [34] and SPK [35] mainly due to surgical complications. However, only morbid obesity $\left(\mathrm{BMI}>40 \mathrm{~kg} / \mathrm{m}^{2}\right.$ ) should be considered as an absolute contraindication, although bariatric surgery before transplantation can ameliorate it [36].

As patients with diabetic nephropathy have increased cardiovascular morbidity and mortality, the pre-transplant evaluation should focus on the presence and the severity of coronary and peripheral artery disease. Although, there is no consensus regarding the optimal protocol for cardiovascular risk stratification, most centers refer the candidates for cardiac stress testing and/or coronary angiography, especially in ages older than 30 years or history of diabetes $>20$ years for T1DM and ages $>55-60$ years for T2DM as well as dyslipidemia, history of smoking and presence of cerebrovascular or peripheral vascular disease [37]. However, this approach has been challenged by the provocative study of Patel et al. which reported that even aggressive pre-transplant testing and coronary interventions did not translate into better outcomes post transplantation in high risk patients $[38,39]$.
Peripheral arterial occlusive disease and carotid arteries stenosis examination by ultrasound are also mandatory in the pre-transplant evaluation. Many centers suggest a more thorough examination in high risk patients by $\mathrm{CT}$ or MR angiography or even intra-arterial angiography $[15,37,40]$.

As pancreas transplantation cannot be considered as an immediate life-saving procedure [10], its possible advantages should be carefully balanced against the potent complications of the surgical procedure and the long-term side-effects of immunosuppression [40,41]. The state of the art approach is to refer the patient with advanced diabetic nephropathy to the transplant centre early, when his estimated Glomerular Filtration Rate (eGFR) is about $25-30 \mathrm{ml} / \mathrm{min}$ in order to provide enough time for evaluation of both the transplant candidate and any potential living donors $[15,38,41]$. However, as most diabetics with CKD are not referred early to a nephrologist, this remains the exemption and not the standard procedure. Nevertheless, by an early referral, the transplant team can evaluate more thoroughly the diabetic candidate and order more complicated investigations such as coronary angiography without increasing risk of premature start of dialysis [38]. In addition, if SPK is not the goal, as in most patients with T2DM, it will also provide time to search for living related kidney transplantation with the most suitable donor or even alternative options for preemptive transplantation in cases of immunologically incompatible but still qualified donors, such as kidney paired donation [15].

Regarding T2DM patients eligible for transplantation, the United Network for Organ Sharing (UNOS) has defined the following criteria for SPK: a) insulin therapy and C-peptide level $<2 \mathrm{ng} / \mathrm{ml}$ or b) insulin therapy with C-peptide level $>2 \mathrm{ng} / \mathrm{ml}$ and $\mathrm{BMI}<28 \mathrm{~kg} / \mathrm{m}^{2}$ [42]. The initial concern regarding pancreas transplantation in T2DM patients was insulin resistance that prevails in this type of DM and may result in lower pancreas allograft survival due to $\beta$ cell exhaustion from the increased insulin demands [43]. Sampaio et al. have analyzed the UNOS database regarding SPK in T1DM and T2DM patients, reporting equal risks of death and kidney or pancreas failure among the two categories after adjustments for other risk factors [44]. However, in the unadjusted analysis older age and longer dialysis vintage were associated with increased mortality in T2DM compared with T1DM recipients. On the contrary, Wiseman and Gralla have recently reported in a retrospective database analysis of 6416 T2DM patients who underwent transplantation from 2000-2008, that there is no clear patient or graft survival advantage for T2DM patients undergoing SPK compared with DDKT, whereas LDKT provided superior outcomes [42]. These results support a more cautious approach for SPK in T2DM patients eligible for transplantation.

\section{Immunosuppressive Protocols}

Immunosuppressive protocols for kidney transplantation have seen a dramatic progress from the first years, when the only weapons of the transplant team were steroids and azathioprine. Calcineurin inhibitors (cyclosporine and tacrolimus) and mycophenolate mofetil (MMF) have eventually reduced acute rejection rates below $10 \%$ and the mammalian target of rapamycin (mTOR) inhibitors (sirolimus and everolimus) have also been used in order to reduce nephrotoxicity and improve long-term outcomes [45]. Recently, belatacept has been officially approved for maintenance immunossupresion in KT [46]. In addition, most centers are using induction therapies with monoclonal or polyclonal antibodies in order to reduce acute rejection during the first post-transplant weeks [47].

Regarding pancreas transplantation, initial high rejection rates 
$(>60 \%)$ of the pancreatic grafts were one of the major obstacles during the cyclosporine era $[7,10]$. However, recent progress in immunosuppression has reduced rejection rates below $25 \%$ or even lower depending on the applied protocols in each center. Induction therapies with monoclonal or polyclonal antibodies have also reduced the incidence and the severity of the rejection episodes [6-11]. Most transplant centers have been using $\mathrm{T}$ cell depleting antibodies (thymoglobulin or alemtuzumab), whereas interleukin-2 receptor antibodies (basiliximab and daclizumab) have shown higher rejection rates and their use is declining $(<10 \%)[11]$.

Regarding maintenance immunosuppression, most centers are now using a combination of tacrolimus and MMF, which has shown superior results compared with older protocols based on cyclosporine or azathioprine. Some centers are also using sirolimus based protocols with good results [48]. Although steroids were considered as crucial components of the immunosuppressive regimen in the first years of pancreas transplantation, more and more centers are now applying steroid avoidance or sparing protocols with excellent results, trying to reduce their detrimental side effects [49-52]. It should also be noted that graft loss in T1DM patients is not always a result of alloimmunity and autoimmune recurrence has been noticed in transplants between identical twins not receiving immunosuppression [22].

Although there is no consensus about the optimal immunosuppressive protocol in pancreas or kidney-pancreas transplantation, most authorities suggest induction therapy with depleting antibodies and maintenance therapies based on tacrolimus, sirolimus or MMF, with early or late steroid withdrawal [6-11].

\section{Allogeneic Islet Transplantation Overview}

Allogeneic islet transplantation in humans became popular after the landmark study of Shapiro et al. the Edmonton group in 2000 [13] which showed insulin independence in seven T1DM patients with a steroid free regimen. Nevertheless, these first encouraging results could not be fully reproduced by other centers and patients needed multiple islet transfusions with a long-term success below 10\% [5355]. In addition, the immunosuppressive protocols are potentially nephrotoxic and may be accompanied with a deterioration of the renal function [56,57], whereas the failed islet grafts may lead to recipients' alloimmunization (sensitization) by the production of de novo antiHLA antibodies in titers ranging between 10.8\%-31\% [56-58]. These poor results have raised skepticism in the transplant community [59] and today only a few centers continue islet transplants on a regular basis [54,55]. The main advantage of islet transplantation is that it is a minimally invasive procedure by the percutaneous infusion of the islets into the portal vein with extremely low complication rates. However, there is a need for life-time immunosuppression and the current protocols have included more intensified regimens (thymoglobulin, alemtuzumab, anti-CD3 monoclonal antibodies, efalizumab, belatacept, etanercept etc.), in order to get better outcomes with islets from single donors [53-55,60]. This approach has been translated into improved long-term outcomes with insulin independence up to $44 \%$ at 3 years after islet transplantation and lower rates of adverse effects [61]. Although the ultimate goal of islet transplantation would be to achieve insulin independence, this can be done rarely and the current goals focus mainly on protection from hypoglycemia, reduction of the daily dose of insulin and correction of HbA1c [54,55]. Due to all the above reasons, islet transplantation should be still considered as an experimental procedure performed only in experienced transplant centers and it is currently recommended only for well informed T1DM patients with difficult to control diabetes and well preserved renal function, or already immunosuppressed T1DM patients undergoing simultaneous islet-kidney transplantation, islet transplantation after SPK and failure of the pancreas graft, or islet transplantation following a successful kidney transplant [54,57]. As there is also a limited pool of donors for pancreata, islet transplantation research is now focusing on alternative resources such as stem cells or xenogeneic (porcine) islets [54,62].

\section{Transplant Options for the Patient with Diabetic Nephropathy: The Need for Individualization}

The above overview of all the possible options for transplantation in patients with diabetic nephropathy emphasizes that as there are no clear indications for each procedure (KT, SPK, PTA, PAK, or IT), the final decision should always be made after careful examination of each diabetic candidate (type of diabetes, comorbidities and life expectancy), his options for living related transplantation, the mean waiting time of every transplant center for cadaveric transplantation and its expertise regarding all these procedures. In addition, patient's preferences and expectations should be thoroughly discussed and not be neglected. In the next paragraphs we will try to provide data regarding possible scenarios of patients with T1DM or T2DM and various degrees of CKD. We have excluded from this discussion T2DM patients with early CKD, as these patients are not considered eligible for any kind of transplantation and the primary care physicians should mainly focus on the delay of CKD progression and the treatment of comorbidities.

\section{The T1DM patient with early stage CKD}

This is the most difficult scenario, as these patients are rather young and have rather preserved kidney function. Here, the dilemma is not focusing primarily on the kidney but on diabetes, its complications and the possible impact of the transplantation procedure on renal function. The patient has three options: a) to remain under insulin therapy and wait for CKD to progress more, b) to be listed for PTA c) to undergo IT. Regarding the last two options, the American Diabetes Association [63] has fostered the following statement: "In the absence of indications for kidney transplantation, pancreas transplantation should only be considered a therapy in patients who exhibit these three criteria: a) a history of frequent, acute, and severe metabolic complications (hypoglycemia, hyperglycemia, ketoacidosis) requiring medical attention; b) clinical and emotional problems with exogenous insulin therapy that are so severe as to be incapacitating; and c) consistent failure of insulin based management to prevent acute complications". The same criteria may also apply for IT "within the setting of controlled research studies" [63]. However, direct comparisons regarding longterm efficacy of IT and PTA can not be made at the present time [64]. Regarding PTA, patients with well preserved eGFR $(80-100 \mathrm{ml} /$ $\mathrm{min} / 1.73 \mathrm{~m}^{2}$ ) are quite unlikely to progress to ESRD, although about $30 \%$ of them may need kidney transplantation after 10 years $[10,17]$. On the contrary, patients with lower eGFR may progress more rapidly after PTA due to the nephrotoxicity of the immunosuppressive medications [65]. The long-term impact of PTA on kidney function has not been extensively studied. The first report form Minnesota indicated reversal of the glomerular lesions in 8 patients after 5-10 years [66] However, creatinine clearance declined significantly, probably due to the loss of the diabetes induced hyperfiltration and the nephrotoxicity of the immunosuppression [66-68]. Slightly better results with less aggressive immunosuppression have also been reported from Italy, but with a shorter follow-up period [64]. Living in the era of evidence based medicine, we have no solid data about long-term outcomes after 
PTA or IT in this group of patients and prospective controlled trials, although designed, have not progressed so far [64]. Regarding IT, the main obstacles are the rather poor long-term results regarding insulin independence, the need for long-term immunosuppression and its risks (infections, malignancies etc.), the possible more rapid deterioration of the renal function due to nephrotoxicity (calcineurin inhibitors) and the risk of alloimmunization [53-58]. However, successful IT has also been associated with significant benefits, such as stabilization or even improvement of retinopathy and neuropathy $[54,55]$.

\section{The T1DM patient with advanced CKD}

In T1DM patients with advanced CKD (eGFR $<50-60 \mathrm{ml} /$ $\min / 1.73 \mathrm{~m}^{2}$ ) the first option should include SPK or pre-emptive KT with PAK $[10,17]$ if is feasible. These patients present extremely high morbidity and mortality, which are multiplied when they start renal replacement therapies $[3,69,70]$. The current goal is to avoid dialysis and search options for pre-emptive KT or SPK $[15,38]$. Timely referral to a transplant center has been shown to increase transplant rates and decrease mortality [15]. Living related SPK from well selected donors remains an option but only for very experienced transplant centers around the world [16,23-25]. Regarding KT, living transplant options (LDKT) should be extensively discussed with the diabetic candidate. The donors' pool should not be limited by HLA matching and other options such as ABO/HLA incompatible donors, good Samaritan donors, or paired donors should also be discussed [15]. Listing for DDKT should be applied when eGFR is less than $20 \mathrm{ml} / \mathrm{min}$. There are also solid data that LDKT does not only improve survival compared with dialysis, but also presents highest short-term survival rates (for both the patient and the allograft) compared not only with DDKT but also with SPK transplants [71]. This is mainly due to the higher rates of surgical complications during SPK, but this advantage starts to become less clear after 5-6 years, when the results favour the SPK procedure $[71,72]$. Based on a thorough review of all the available data, Wiseman [9] has recently concluded that LDKT is without doubt superior to DDKT and probably to SPK, if we consider that about $10-15 \%$ of the SPK patients may lose the pancreas allograft during the first year. However, for the remaining $85 \%$ of the SPK recipients, SPK is superior to LDKT.

Another issue that needs to be addressed is what to do with a patient after a successful LDKT or DDKT. Should he proceed with PAK transplantation, or remain a diabetic with a functioning kidney, but with all the problems of hyperglycemia? The potential risks of the operation for PAK transplantation can not be ignored and some older studies had reported a 3-4 fold increase of death risk during the first three months $[73,74]$. In addition, pancreas graft survival in PAK is inferior to SPK transplantation due to higher rejection rates and it has decreased as a transplant option by $50 \%$ from 2004 to 2010 [11]. However, some studies have reported more optimistic results with more aggressive immunosuppressive protocols [75].

Regarding IT in this population, there is no rational for the procedure, unless it is accompanied by or following kidney transplantation [54]. However, the accumulated experience is extremely limited at the moment [57].

\section{The T2DM patient with advanced CKD}

T2DM patients dominate by far (95\%) among patients with CKD diagnosed with diabetic nephropathy, are usually older than T1DM patients and carry significant comorbidities, which may be contraindications for transplantation. However, current data support the concept that transplantation offers them a survival advantage over dialysis [3]. So, all attempts should focus on pre-emptive KT, but even SPK transplantation may be offered in selected T2DM patients [43,7678]. According to the 2011 IPTR report about $8 \%$ of SPK, $5 \%$ of PAK and $1 \%$ of PTA were performed in T2DM patients [11]. These patients were mainly males $(67 \%)$ and presented higher BMI compared with T1DM patients [11]. As data regarding long-term survival after SPK are rather conflicting $[42,44]$, most authorities $[42,43,78]$ suggest that the best option would be to target for LDKT and consider SPK only in well selected cases with a metabolic profile similar to T1DM. In addition, recent data from bariatric surgery implicate a more favorable outcome for T2DM patients with diabetic nephropathy and BMI $>32$ $\mathrm{kg} / \mathrm{m}^{2}$ before KT [78-80]. However, it is worth noting that T2DM patients have inferior outcomes after KT compared with non diabetics and present more early and late complications, mainly due to the detrimental effects of hyperglycemia, which may be aggravated by the immunosuppressive regimens $[76,77,81,82]$.

\section{Conclusions}

Recent progress in surgery and immunosuppression has expanded the "transplant menu" for patients with diabetic nephropathy.

As pre-emptive transplantation provides a clear survival advantage over dialysis, all diabetic patients with CKD should be referred for early evaluation by a transplant center.

For T1DM patients, LDKT and SPK transplantation offer superior and approximately equivalent long-term patient and renal allograft survival. PAK transplant rates tend to decline due to surgical and immunological complications, but it may still be considered for well selected candidates with preserved kidney allograft function.

For T2DM patients, not only LDKT, but even DDKT are superior to dialysis. SPK transplantation should be offered only in selected cases with special metabolic characteristics similar to T1DM.

PTA should be considered only for selected cases of T1DM with well preserved renal function (eGFR $>80 \mathrm{ml} / \mathrm{min} / 1.73 \mathrm{~m}^{2}$ and minimal proteinuria) as it is related with rapid deterioration of renal function.

Islet transplantation should still be considered as an experimental procedure for T1DM, and has no place at the moment in patients with advanced CKD, but it may be applied in already immunosuppressed patients following KT. Nevertheless, a lot of progress regarding this field is anticipated in the near future.

However, the most appropriate transplant option for patients with diabetic nephropathy therapy should always be individualized, taking under consideration the patients' preferences and expectations, their overall medical condition and the transplant center's experience with all these procedures.

\section{References}

1. Rossing P (2006) Diabetic nephropathy: worldwide epidemic and effects of current treatment on natural history. Curr Diab Rep 6: 479-483.

2. Patterson CC, Dahlquist GG, Gyürüs E, Green A, Soltész G; EURODIAB Study Group (2009) Incidence trends for childhood type 1 diabetes in Europe during 1989-2003 and predicted new cases 2005-20: a multicentre prospective registration study. Lancet 373: 2027-2033.

3. Wolfe RA, Ashby VB, Milford EL, Ojo AO, Ettenger RE, et al. (1999) Comparison of mortality in all patients on dialysis, patients on dialysis awaiting transplantation, and recipients of a first cadaveric transplant. $\mathrm{N}$ Engl $\mathrm{J}$ Med 341: $1725-1730$

4. Kelly WD, Lillehei RC, Merkel FK, Idezuki Y, Goetz FC (1967) Allotransplantation of the pancreas and duodenum along with the kidney in diabetic nephropathy. Surgery 61: 827-837. 
Citation: Fourtounas C, Dousdampanis P (2013) Kidney, Pancreas and Islet Transplant Options for Patients with Diabetic Nephropathy. J Diabetes Metab S9: 001. doi:10.4172/2155-6156.S9-001

Page 6 of 7

5. Boggi U, Amorese G, Marchetti P (2010) Surgical techniques for pancreas transplantation. Curr Opin Organ Transplant 15: 102-111.

6. Boggi U, Vistoli F, Egidi FM, Marchetti P, De Lio N, et al. (2012) Transplantation of the pancreas. Curr Diab Rep 12: 568-579.

7. Gruessner AC, Sutherland DE, Gruessner RW (2010) Pancreas transplantation in the United States: a review. Curr Opin Organ Transplant 15: 93-101.

8. Richter A, Lerner S, Schröppel B (2011) Current state of combined kidney and pancreas transplantation. Blood Purif 31: 96-101.

9. Wiseman AC (2010) The role of kidney-pancreas transplantation in diabetic kidney disease. Curr Diab Rep 10: 385-391.

10. White SA, Shaw JA, Sutherland DE (2009) Pancreas transplantation. Lance 373: $1808-1817$

11. Gruessner AC (2011) 2011 update on pancreas transplantation: comprehensive trend analysis of 25,000 cases followed up over the course of twenty-four years at the International Pancreas Transplant Registry (IPTR). Rev Diabet Stud 8 :

12. Scharp DW, Lacy PE, Santiago JV, McCullough CS, Weide LG, et al. (1990) Insulin independence after islet transplantation into type I diabetic patient. Diabetes 39: 515-518.

13. Shapiro AM, Lakey JR, Ryan EA, Korbutt GS, Toth E, et al. (2000) Islet transplantation in seven patients with type 1 diabetes mellitus using a glucocorticoid-free immunosuppressive regimen. N Engl J Med 343: 230-238.

14. Becker BN, Rush SH, Dykstra DM, Becker YT, Port FK (2006) Preemptive transplantation for patients with diabetes-related kidney disease. Arch Intern Med 166: 44-48.

15. Pavlakis M, Kher A (2012) Pre-emptive kidney transplantation to improve survival in patients with type 1 diabetes and imminent risk of ESRD. Semin Nephrol 32: 505-511.

16. Sutherland DE, Radosevich D, Gruessner R, Gruessner A, Kandaswamy R (2012) Pushing the envelope: living donor pancreas transplantation. Curr Opin Organ Transplant 17: 106-115.

17. Sutherland DE, Gruessner RW, Dunn DL, Matas AJ, Humar A, et al. (2001) Lessons learned from more than 1,000 pancreas transplants at a single institution. Ann Surg 233: 463-501.

18. Scalea JR, Cooper M (2012) Current concepts in the simultaneous transplantation of kidney and pancreas. J Intensive Care Med 27: 199-206.

19. Tan M, Kandaswamy R, Sutherland DE, Gruessner RW (2005) Laparoscopic donor distal pancreatectomy for living donor pancreas and pancreas-kidney transplantation. Am J Transplant 5: 1966-1970.

20. Boggi U, Signori S, Vistoli F, D'Imporzano S, Amorese G, et al. (2012) Laparoscopic robot-assisted pancreas transplantation: first world experience. Transplantation 93: 201-206.

21. Boggi U, Amorese G, Marchetti P, Mosca F (2011) Segmental live donor pancreas transplantation: review and critique of rationale, outcomes, and current recommendations. Clin Transplant 25: 4-12.

22. Sutherland DE, Sibley R, Xu XZ, Michael A, Srikanta AM, et al. (1984) Twin-totwin pancreas transplantation: reversal and reenactment of the pathogenesis of type I diabetes. Trans Assoc Am Physicians 97: 80-87.

23. Gruessner RW, Sutherland DE (1996) Simultaneous kidney and segmental pancreas transplants from living related donors - the first two successful cases. Transplantation 61: 1265-1268.

24. Gruessner RW, Kendall DM, Drangstveit MB, Gruessner AC, Sutherland DE (1997) Simultaneous pancreas-kidney transplantation from live donors. Ann Surg 226: 471-480.

25. Zielinski A, Nazarewski S, Bogetti D, Sileri P, Testa G, et al. (2003) Simultaneous pancreas-kidney transplant from living related donor: a singlecenter experience. Transplantation 76: 547-552.

26. Serón D, Fulladosa X, Moreso $F(2005)$ Risk factors associated with the deterioration of renal function after kidney transplantation. Kidney Int Suppl S113-117.

27. Ramos E, Aoun S, Harmon WE (2002) Expanding the donor pool: effect on graft outcome. J Am Soc Nephrol 13: 2590-2599.
28. Pascual J, Zamora J, Pirsch JD (2008) A systematic review of kidney transplantation from expanded criteria donors. Am J Kidney Dis 52: 553-586.

29. Michalak G, Kwiatkowski A, Czerwinski J, Chmura A, Lisik W, et al. (2003) Simultaneous pancreas-kidney transplantation: analysis of donor factors. Transplant Proc 35: 2337-2338.

30. Kasiske BL, Cangro CB, Hariharan S, Hricik DE, Kerman RH, et al. (2001) The evaluation of renal transplantation candidates: clinical practice guidelines. Am J Transplant 1: 3-95

31. EBPG (European Expert Group on Renal Transplantation); European Rena Association (ERA-EDTA); European Society for Organ Transplantation (ESOT) (2000) European Best Practice Guidelines for Renal Transplantation (part 1) Nephrol Dial Transplant 15: 1-85

32. Dousdampanis $P$, Trigka K, Fourtounas C (2012) Diagnosis and management of chronic kidney disease in the elderly: a field of ongoing debate. Aging Dis 3: $360-372$.

33. Afaneh C, Rich BS, Aull MJ, Hartono C, Leeser DB, et al. (2011) Pancreas transplantation: does age increase morbidity? J Transplant 2011: 596801.

34. Meier-Kriesche HU, Arndorfer JA, Kaplan B (2002) The impact of body mass index on renal transplant outcomes: a significant independent risk factor fo graft failure and patient death. Transplantation 73: 70-74.

35. Sampaio MS, Reddy PN, Kuo HT, Poommipanit N, Cho YW, et al. (2010) Obesity was associated with inferior outcomes in simultaneous pancreas kidney transplant. Transplantation 89: 1117-1125.

36. Porubsky M, Powelson JA, Selzer DJ, Mujtaba MA, Taber T, et al. (2012) Pancreas transplantation after bariatric surgery. Clin Transplant 26: E1-6.

37. Kasiske BL, Israni AK, Snyder JJ, Camarena A; COST Investigators (2011) Design considerations and feasibility for a clinical trial to examine coronary screening before kidney transplantation (COST). Am J Kidney Dis 57: 908-916

38. Gaston RS, Basadonna G, Cosio FG, Davis CL, Kasiske BL, et al. (2004) Transplantation in the diabetic patient with advanced chronic kidney disease: a task force report. Am J Kidney Dis 44: 529-542.

39. Patel RK, Mark PB, Johnston N, McGeoch R, Lindsay M, et al. (2008) Prognostic value of cardiovascular screening in potential renal transplant recipients: a single-center prospective observational study. Am J Transplant 8: 1673-1683.

40. Eller K, Kniepeiss D, Rosenkranz AR (2013) Preoperative risk evaluation: where is the limit for recipients of a pancreatic graft? Curr Opin Organ Transplant 18 97-101.

41. Wiseman AC (2009) Simultaneous pancreas kidney transplantation: a critica appraisal of the risks and benefits compared with other treatment alternatives. Adv Chronic Kidney Dis 16: 278-287.

42. Wiseman AC, Gralla J (2012) Simultaneous pancreas kidney transplant versus other kidney transplant options in patients with type 2 diabetes. Clin J Am Soc Nephrol 7: 656-664.

43. Cohen DJ, Ratner LE (2012) Type 2 diabetes: the best transplant option is stil uncertain. Clin J Am Soc Nephrol 7: 530-532.

44. Sampaio MS, Kuo HT, Bunnapradist S (2011) Outcomes of simultaneous pancreas-kidney transplantation in type 2 diabetic recipients. Clin J Am Soc Nephrol 6: 1198-1206.

45. Webber A, Hirose R, Vincenti F (2011) Novel strategies in immunosuppression issues in perspective. Transplantation 91: 1057-1064.

46. Wekerle T, Grinyó JM (2012) Belatacept: from rational design to clinical application. Transpl Int 25: 139-150.

47. Wagner SJ, Brennan DC (2012) Induction therapy in renal transplant recipients: how convincing is the current evidence? Drugs 72: 671-683.

48. Ciancio G, Sageshima J, Chen L, Gaynor JJ, Hanson L, et al. (2012) Advantage of rapamycin over mycophenolate mofetil when used with tacrolimus for simultaneous pancreas kidney transplants: randomized, single-center trial at 10 years. Am J Transplant 12: 3363-3376.

49. Cantarovich D, Karam G, Hourmant M, Dantal J, Blancho G, et al. (2005) Steroid avoidance versus steroid withdrawal after simultaneous pancreaskidney transplantation. Am J Transplant 5: 1332-1338.

50. Cantarovich D, Giral-Classe M, Hourmant M, Dantal J, Blancho G, et al. (2000) Low incidence of kidney rejection after simultaneous kidney-pancreas 
Citation: Fourtounas C, Dousdampanis P (2013) Kidney, Pancreas and Islet Transplant Options for Patients with Diabetic Nephropathy. J Diabetes Metab S9: 001. doi:10.4172/2155-6156.S9-001

transplantation after antithymocyte globulin induction and in the absence of corticosteroids: results of a prospective pilot study in 28 consecutive cases. Transplantation 69: 1505-1508.

51. Cantarovich D, Vistoli F (2009) Minimization protocols in pancreas transplantation. Transpl Int 22: 61-68.

52. Kimelman M, Brandacher G (2013) Trends in immunosuppression after pancreas transplantation: what is in the pipeline? Curr Opin Organ Transplant 18: $76-82$

53. Ludwig B, Ludwig S, Steffen A, Saeger HD, Bornstein SR (2010) Islet versus pancreas transplantation in type 1 diabetes: competitive or complementary? Curr Diab Rep 10: 506-511.

54. Shapiro AM (2011) State of the art of clinical islet transplantation and nove protocols of immunosuppression. Curr Diab Rep 11: 345-354

55. Vardanyan M, Parkin E, Gruessner C, Rodriguez Rilo HL (2010) Pancreas vs. islet transplantation: a call on the future. Curr Opin Organ Transplant 15: 124 130.

56. Senior PA, Zeman M, Paty BW, Ryan EA, Shapiro AM (2007) Changes in renal function after clinical islet transplantation: four-year observational study. Am J Transplant 7: 91-98.

57. Ferrari-Lacraz S, Berney T, Morel P, Marangon N, Hadaya K, et al. (2008) Low risk of anti-human leukocyte antigen antibody sensitization after combined kidney and islet transplantation. Transplantation 86: 357-359.

58. Cantarovich D, Vistoli F, Bignon JD (2008) Anti-human leukocyte antigen antibodies after islet transplantation: what do they really mean? Transplantation 86: 204-205.

59. Bromberg JS, LeRoith D (2006) Diabetes cure--is the glass half full? N Engl J Med 355: 1372-1374.

60. Bellin MD, Barton FB, Heitman A, Harmon JV, Kandaswamy R, et al. (2012) Potent induction immunotherapy promotes long-term insulin independence after islet transplantation in type 1 diabetes. Am J Transplant 12: 1576-1583.

61. Barton FB, Rickels MR, Alejandro R, Hering BJ, Wease S, et al. (2012) Improvement in outcomes of clinical islet transplantation: 1999-2010. Diabetes Care 35: 1436-1445

62. Dufrane D, Gianello P (2012) Pig islet for xenotransplantation in human: structural and physiological compatibility for human clinical application. Transplant Rev (Orlando) 26: 183-188.

63. Robertson RP, Davis C, Larsen J, Stratta R, Sutherland DE; American Diabetes Association (2006) Pancreas and islet transplantation in type 1 diabetes. Diabetes Care 29: 935.

64. Cantarovich D, Perrone $\vee$ (2012) Pancreas transplant as treatment to arrest renal function decline in patients with type 1 diabetes and proteinuria. Semin Nephrol 32: 432-436

65. Scalea JR, Butler CC, Munivenkatappa RB, Nogueira JM, Campos L, et al. (2008) Pancreas transplant alone as an independent risk factor for the development of renal failure: a retrospective study. Transplantation 86: 17891794.
66. Fioretto P, Steffes MW, Sutherland DE, Goetz FC, Mauer M (1998) Reversal of lesions of diabetic nephropathy after pancreas transplantation. N Engl J Med 339: 69-75.

67. Fioretto P, Najafian B, Sutherland DE, Mauer M (2011) Tacrolimus and cyclosporine nephrotoxicity in native kidneys of pancreas transplant recipients. Clin J Am Soc Nephrol 6: 101-106.

68. Fioretto P, Sutherland DE, Najafian B, Mauer M (2006) Remodeling of rena interstitial and tubular lesions in pancreas transplant recipients. Kidney Int 69 : 907-912.

69. Rosolowsky ET, Skupien J, Smiles AM, Niewczas M, Roshan B, et al. (2011) Risk for ESRD in type 1 diabetes remains high despite renoprotection. J Am Soc Nephrol 22: 545-553.

70. Forsblom C, Harjutsalo V, Thorn LM, Wadén J, Tolonen N, et al. (2011) Competing-risk analysis of ESRD and death among patients with type diabetes and macroalbuminuria. J Am Soc Nephrol 22: 537-544.

71. Young BY, Gill J, Huang E, Takemoto SK, Anastasi B, et al. (2009) Living donor kidney versus simultaneous pancreas-kidney transplant in type I diabetics: an analysis of the OPTN/UNOS database. Clin J Am Soc Nephrol 4: 845-852.

72. Morath C, Zeier M, Döhler B, Schmidt J, Nawroth PP, et al. (2010) Transplantation of the type 1 diabetic patient: the long-term benefit of a functioning pancreas allograft. Clin J Am Soc Nephrol 5: 549-552.

73. Venstrom JM, McBride MA, Rother KI, Hirshberg B, Orchard TJ, et al. (2003) Survival after pancreas transplantation in patients with diabetes and preserved kidney function. JAMA 290: 2817-2823.

74. Gruessner RW, Sutherland DE, Gruessner AC (2004) Mortality assessment fo pancreas transplants. Am J Transplant 4: 2018-2026.

75. Fridell JA, Mangus RS, Hollinger EF, Taber TE, Goble ML, et al. (2009) The case for pancreas after kidney transplantation. Clin Transplant 23: 447-453.

76. Luan FL, Samaniego M (2010) Transplantation in diabetic kidney failure patients: modalities, outcomes, and clinical management. Semin Dial 23: 198 205.

77. Guerra G, llahe A, Ciancio G (2012) Diabetes and kidney transplantation: past present, and future. Curr Diab Rep 12: 597-603.

78. Sener A, Cooper M, Bartlett ST (2010) Is there a role for pancreas transplantation in type 2 diabetes mellitus? Transplantation 90: 121-123.

79. Koshy AN, Coombes JS, Wilkinson S, Fassett RG (2008) Laparoscopic gastric banding surgery performed in obese dialysis patients prior to kidney transplantation. Am J Kidney Dis 52: e15-17

80. Scalea JR, Cooper M (2012) Surgical strategies for type II diabetes. Transplan Rev (Orlando) 26: 177-182.

81. Cosio FG, Hickson LJ, Griffin MD, Stegall MD, Kudva Y (2008) Patient survival and cardiovascular risk after kidney transplantation: the challenge of diabetes. Am J Transplant 8: 593-599.

82. Wiesbauer F, Heinze G, Regele H, Hörl WH, Schernthaner GH, et al. (2010) Glucose control is associated with patient survival in diabetic patients after renal transplantation. Transplantation 89: 612-619. 